\title{
TWO-PROCESS THEORY OF PERCEIVED CONTROL
}

\section{Changing the workspace and changing the self}

\author{
Daibin Xie*
}

\section{Background}

The two-process theory of perceived control (TTPC) was proposed by Rothbaum, Weisz and Snyder (1982). The theory incorporates cognitive development ideas (Piaget, 1970) and suggests that a feeling of control can be gained by two means - changing the world and changing the self. The two processes are often intertwined but prioritised differently according to circumstances. Since its introduction, the theory has attracted great attention in the field of psychological study as it challenges traditional Western understanding about control (Morling \& Evered, 2006).

Control has long been regarded as an important construct affecting people's psychological functioning (Skinner, 1996). Psychologists believe that humans have a universal desire to exert control over their environments or make choices in order to produce behaviour-event contingency (Heckhausen \& Schulz, 1995). A lack of control has been associated with learned helplessness (Seligman, 1975), self-denial (DeCharms, 1979), defects in 'self-efficacy' (Bandura, 1977) and negative emotions such as dissatisfaction, anxiety, depression and even anger (Mirowsky \& Ross, 1990). Because of this importance, control variables, in one form or another, underlie much theorising of environmental and organisational psychology. For example, Rotter's (1966) locus of control theory considers the person's beliefs about control as a key driver of behaviours, suggesting that those people who see external forces as controlling outcomes (external locus of control) are more likely to manifest inward behaviours such as passivity, withdrawal, submissiveness and depression than are those who believe in their own effort and ability to change the environment as controlling outcomes (internal locus of control). Seligman's (1975) learned helplessness model focuses on the effect of objective contingency between action and outcome on control agents (the individuals or groups who exert the control) and maintains that prolonged exposure to the situation failing to influence the environment produces cognitive, motivational and emotional deficits (Seligman, 1975). Karasek's (1979) job demands/control model (see Chapter 3 The Job Demands-Resources Model) sees the interaction of job demands and latitude as a determining factor of worker well-being.

The aforementioned three theories are often cited in social or occupational stress studies due to their lucid account of maladaptive behaviours. In each case individuals' ability to affect the environment or make choices/decisions is emphasised and inward behaviours are *Corresponding author: daibin.xie.13@alumni.ucl.ac.uk 
linked to relinquished control. However, Rothbaum et al. (1982) argued that those classical control theories have overlooked the important effect of cognitive mediation. According to cognitive development theorists, the goal of development is equilibration, involving assimilation and accommodation; both reflect strategies to optimise the individual's adaptation to the environment (Piaget, 1970; Rothbaum et al., 1982). As such, it is possible that perceived control increases when an individual is able to accommodate to reality (Averill, 1973).

Based on the assumption, Rothbaum et al. (1982) suggested that in addition to attempts to influence the environment and the abandonment of action, there is a third alternative - people sometimes flexibly adjust themselves to fit in with the existing reality. For instance, an individual may submerge self-sense in order to foster a sense of belonging at workplaces; some people lower their expectations to avoid disappointment when a task is perceived as difficult or impossible to finish. These responses signal a purpose to maintain perception of control in the form of enhancing group recognition or proactively managing negative effects. In essence, they have a positive nature.

Rothbaum et al. (1982) labelled processes of changing the environment to fit personal needs as primary control and attempts to align themselves with existing realities as secondary control. A key distinguishing characteristic of them is that actions are directed outward to the environment in primary control (e.g. personalise one's workstations) but inward to the individual's cognition in secondary control (e.g. accept having no assigned desk at work). According to Rothbaum et al. (1982), there are four types of secondary control. When people attribute outcomes to chances, for example, linking business success and failure to good or back luck, they may gain illusory control to enhance acceptance of their own fate. Vicarious control is sought when people attempt to create association or to sustain alignment with powerful others such as managers or supervisors to derive feelings of self-esteem. When people attribute negative outcomes to low ability or external constraints, for instance one's status within an organisation, they predict future outcomes and then adjust their expectations to reduce disappointments, which leads to predictive control. Finally, attempts to gain sense of meaning and understanding about existing realities may foster interpretive control (e.g. seeing life suffering as atonement).

The theory views the interaction between people and environment as a dynamic negotiation process involving both assimilation and accommodation. Rothbaum et al. (1982) argued that primary control and secondary control have similar functional values, although secondary control is often used as a back-up response to minimise losses and negative effects after efforts to exert primary control have been ineffective. Heckhausen and Schulz (1995, p. 286) further suggest, "secondary control not only helps in dealing with failures, but also fosters primary control directly by managing its selectivity." Although the primacy of primary control is often questioned by other researchers, the theory has opened a new window for psychological study about control. It has inspired a number of later researchers to derive new findings and develop new hypotheses.

For example, Heckhausen and Schulz (1995) developed a life-span theory of development based on the concept of primary and secondary control. They suggest, "trade-offs between primary and secondary control undergo systematic shifts across the life course in response to the opportunities and constraints encountered" (Heckhausen \& Schulz, 1995, p. 284). As people age, they are more able to regulate their emotions and thereby increase secondary control ability (Heckhausen \& Schulz, 1995; McConatha \& Huba, 1999).

Cross-cultural studies reported also that the trade-off between primary control and secondary control is culturally conditioned. It is suggested that, generally, East Asians show a stronger tendency for secondary control then do Americans or Europeans (Kim \& Markus, 1999; Peng \& Nisbett, 1999; Spector, Sanchez, Siu, Salgado, \& Ma, 2004; Weisz, Rothbaum, \& Blackburn, 1984). Leung (2001) elaborated that workers in collectivistic cultures are more unconditionally 
benevolent and are more likely to sacrifice certain personal interest to enhance in-group welfare to get group recognition.

\section{Applicability to workplace studies}

The control issue also resonates in the field of workplace research. In the literature, much has been written about the benefits of fulfilling user needs and providing more user control over the work environment. A core argument of workplace researchers is that the increase of perceived control brought about by more flexibility and more user engagement in workplace design and management can enhance employees' sense of belonging and ownership at the workplace and enable them to create an environment better fitting their task needs and personal expectations (Vischer, 2008).

Control variables often examined in the workplace literature are control over indoor environment qualities (e.g. ventilation, lighting, noise and temperature), personalisation, layout adjustability and flexibility, visual access, acoustic privacy, adjustable furniture, choice of time and location of work, user engagement in workplace design and ergonomics training to improve control (Huang, Robertson, \& Chang, 2004; Leaman, 1995; O’Neill, 2010; O’Neill \& Carayon, 1993; Robertson \& O’Neill, 1999; Veitch \& Newsham, 2000; Vischer, 2008). Vischer (2008) theorised that, in general, workplace control manifests itself in two layers: mechanical control and empowerment. Mechanical control involves direct behaviours changing the physical workplace settings such as lighting, temperature, acoustics, windows, furnishing, office layout, displays, personalisation, time and location of work, etc. Empowerment involves actions to increase opportunities for employees to participate in workspace decision-making.

The problem is, for most workplace users, they are rarely able to engage in the workplace design process or change the physical workplace optimally to suit them; rather, what they can do is accept what they find as 'givens' (Leaman, 2003). But ironically, this does not stop office workers continuously committing themselves to an undesirable workplace. Why?

According to the theoretical proposition of TTPC, it is reasonable to argue that some forms of positive effect have been caused by those self-adaptive behaviours. Telling evidence is found in the case study of Leaman (1995). It shows that in post-occupancy surveys in UK, offices rated as 'good' often are not those with the best physical conditions but are those with the highest forgiveness ratings. The measure 'forgiveness' is calculated by dividing the overall comfort/ satisfaction of occupants with the mean of measured workplace variables, reflecting occupants' tolerance level on space shortcomings. Leaman (1995, p. 18) explained that workplaces are total systems that consist of human systems and physical systems, and "where these systems correspond closest, there is a greater likelihood that the building will work well in total." Because of this, people are likely to forgive deficiencies and thus maintain a high overall workplace satisfaction when they know every effort has been made but there are difficulties in solving the problems (Leaman, 1995). Echoing Leaman (1995), Xie (2019) empirically found that office workers' workplace expectations are affected by workplace settings. When facing undesirable workplace conditions, office workers may accept the reality and lower their expectations, for instance, depressing the need for privacy in open-planning offices. Or they adjust their expectations and look for compensation to restore a perception of control, for example, by expecting to have more flexibility when the workstation is close to the supervisor. By contrast, the fulfilment of some needs may shift the attention of workspace users and make them forgive other shortcomings; or it may reinforce workplace users' brief of primary control and lead them to raise their work-life standard (Xie, 2019). 
These two examples clearly show the important influence of self-adaptation at workplaces. However, space users' self-adaptation has been largely overlooked by preceding researchers despite its importance and, so far, little is known about it.

The two-process theory of perceived control may be borrowed to fill the research gap as it provides a holistic view in examining the mutual influence between people and the physical work environment. Most importantly, the TTPC offers a dynamic perspective to view the role of workplace needs and control. In literature, various workplace researchers have emphasised that it is important to fulfil user needs and expectations in order to improve workplace satisfaction, productivity and well-being. The idea clearly reflects the mindset of primary control. But according to the TTPC, unfulfilment of needs and expectations does not necessarily lead to dissatisfaction or stress, as people may adapt their needs and expectations according to the context when they are unable to change the fixed physical settings. It appears that office workers' self-adaptation is coined to attempt creating or changing the workplace to fit user needs. Following the logic, researchers may rethink the relationship between workplace control and stress. Lacking personal control on the environment has been regarded as a stressor (McCoy \& Evans, 2005). Obviously, this relationship is doubtful from the standpoint of secondary control, because one of the main functions of secondary control is to help individuals avoid negative influences.

The TTPC stresses the importance of understanding individuals' adaptive patterns and underlying motivations in order to develop proper strategies to help individuals gain or restore a sense of control. The four types of secondary control presented by Rothbaum et al. (1982) provide guidance to crack the nut. They are applicable in workplace research and practice too. For instance, the example that office workers forgive the shortcomings of the workplace after knowing the difficulty to resolve them (Leaman, 1995) can be seen as a practice of predictive control. Following these patterns, researchers and practitioners are able to develop tools to test, predict and manage the outcomes of workplace provisions. For instance, in projects adopting innovation workplace concepts, for example activity-based working, change management agents can foster interpretive control by communicating the benefits of the new design to the company when they know it is an effective strategy to overcome office workers' resistance.

Another important finding of TTPC theorists is the cultural differences in the preferences for secondary control. According to the literature, secondary control appears to be more relevant and preferred in non-Western cultures (Weisz et al., 1984). In other words, direct control over the environment and empowerment may be less expected in those cultures (Fock, Hui, Au, \& Bond, 2013; Zhang \& Begley, 2011). This cultural difference may inspire workplace researchers to rethink the values of mechanical control and empowerment in a cross-cultural context.

Similarly, according to Heckhausen and Schulz (1995), from a life-span development perspective, age and experience play important roles in individuals' choices between primary control and secondary control. Relevant research findings and methodologies may feed workplace research relating to age and generational differences.

\section{Methodology/research approach}

The concept of 'contingency' is central in various control theories, including the TTPC. Setting out from this stance, psychological experiments to test individuals' reactions were widely deployed by early control researchers (Rothbaum et al., 1982). But fieldwork has received increasing attention in recent decades, especially in the field of organisational psychology. Unlike experimental studies in laboratories, individuals' mental schemes and psychological reactions to the environment are difficult to be recorded through well-designed props such as cards games or drawings in fieldwork. Therefore, questionnaire surveys are commonly used to capture 
respondents' psychological action and reaction in field studies with a TTPC viewpoint. Another benefit of using questionnaires is that it enables the quantitative analysis of psychological actions, whereas most experimental psychology studies are qualitative. The following measures are often incorporated in the study of organisational control.

- Work locus of control. The measure assesses briefs about internal/external control in the workplace. For instance, the Work Locus of Control Scale (Spector, 1988) measures whether the respondent believes he or she can control rewards at work through his or her own action. The result may indicate the extent to which the individual stresses primary control.

- Secondary control. Scales are often developed based on the four components of secondary control described by Rothbaum et al. (1982) to reflect respondents' adaptive patterns. For instance, a scale testing the preference for predictive control asks, 'do you agree that you would avoid accepting a task if you think it is overly difficult?'

- Job stressors. In organisational psychology, job stressors measured often are ambiguity of job design, role conflict, interpersonal conflict and lack of autonomy (Spector et al., 2004).

- Strains. For example, job/life dissatisfaction, work anxiety and resign intention.

These measures can be selectively borrowed in studies of workplace control to reflect the individual's motivation of control. But they are clearly insufficient for workplace studies, as workplace is a holistic system consisting of people and physical space. Workplace researchers need to further investigate physical workplace settings and workplace users' needs and expectations with multiple methodologies. Figure 11.1 describes a general process of a workplace study aiming to investigate space users' adaptive behaviours with a TTPC standpoint.

One-way analysis of variance (ANOVA) is often used in comparative studies of control briefs (Spector et al., 2004). Similar methodologies have been used by workplace researchers to investigate the differences in workplace needs and satisfaction between different groups. For instance, Langston, Song and Purdey (2008) examined the occupational differences in workplace satisfaction with a Kruskal-Wallis test (this test is usually used when a measurement variable does not meet the normality assumption of ANOVA). In studies from the standpoint of TTPC, the methodology is also useful for investigating changes in workplace expectations, for example, pre- and post-relocation comparisons.

For TTPC studies, an important task is to understand the effect of external constraints and personal traits (locus of control and preferences for secondary control) on adaptive behaviours. Correlation tests therefore are a powerful tool to conduct the investigation. Some highly interesting research findings have been generated by researchers through the approach, for example the aforementioned adaptive patterns posited by Xie (2019).

In general, the TTPC provides a valuable framework to decipher the complex interaction between people and the environment. For workplace researchers, four research topics in the context of control theory are worth exploring in the future. The first is the effects of workplace settings on workplace users' adaptive behaviours. The second is the comparative study of control preference between cultures, genders and different social groups. The third is the life-span development of control at workplaces - how age and experience affect the trade-off between primary control and secondary control. The final one is the long-term effect of secondary control at workplaces. People may temporarily accept or tolerate a workplace shortcoming, but how long they can tolerate it would be another issue. To address these research topics, researchers need to design their research methodologies carefully to measure office workers' psychology changes, to collect and compare data from different sample groups, and to enable longitudinal studies. 
Step 1. Identify research questions and develop research methodologies based on the research interests

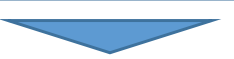

Step 2. Fieldworks: questionnaire survey / site tour to code workspace characteristics

\section{Step 3. Know the facts through descriptive data analysis}

- Identify the locus of control briefs and psychological adaptive patterns of respondents.

- Identify workplace needs / expectations and trace their changes / differences in different contexts.

- Trace changes occupants exert on the physical environment.

\section{Step 4. Get insights through interpretive data analysis}

- Examine the association between psychological adaptations and environmental settings and test related hypothesis.

- Examine the association between primary / secondary control patterns and outcomes of space / workplace expectations, and test related hypothesis.

\section{Step 5. Towards a user-cantred workplace}

Inform workplace design and management practice with insights about what occupants want, what they can possibly give up, how space provisions and deficits would affect them and how to manage their expectations.

Figure 11.1 Process of workplace studies guided with TTPC

\section{Limitation}

The two-process theory of perceived control has attracted great attention in psychology since its introduction because it counterintuitively frames maladaptive behaviours in positive ways and makes reasonable a variety of behaviours that seem maladaptive "from the perspective of an autonomously functioning Western person" (Morling \& Evered, 2006, p. 269). But at the same time, the theory is often faulted for its 'theoretical ambiguity' in regard to the primacy of primary control and the actual function of secondary control and inconsistent findings reported by later researchers. This to some extent would weaken the value of using the theory to interpret self-adaptive behaviours at workplaces. 
In addition, the theory mainly focuses on inward behaviours such as passivity and submissiveness and sees them as psychological strategies to fit in with the environment. Yet, changes of expectations associated with successful delivery of primary control are not addressed.

\section{Theory relevance to practice}

The two-process theory of perceived control as a framework sees the process of people trying to fit in with the environment as a negotiation process between the person and the environment involving assimilating and compromise. It 'vividly' mirrors what is happening in real practice. Insights and knowledge generated from workplace studies guided by the theory may allow workplace designers and managers to better understand what occupants want and what they can compromise on and to predict the effects of different workspace provision scenarios in order to make proper arrangements to better balance the needs of different stakeholders. These findings are also valuable for change management as they may inform change agents to develop more effective communication strategies and design more customised change roadmaps. This is especially important for the implementation of innovation workplace designs and policies such as flexible working, as one main challenge threatening workplace innovation is user resistance.

\section{Further reading}

- Morling, B., \& Evered, S. (2006). Secondary control reviewed and defined. Psychological Bulletin, 132(2), 269-296. https://doi.org/10.1037/0033-2909.132.2.269

- Rothbaum, F., Weisz, J. R., \& Snyder, S. S. (1982). Changing the world and changing the self: A two-process model of perception control. Journal of Personality and Social Psychology, 42, 5-37. https://doi.org/10.1037/0022-3514.42.1.5

- Skinner, E. A. (1996). A guide to constructs of control. Journal of Personality \& Social Psychology, 71(3), 549-570. https://doi.org/10.1037/0022-3514.71.3.549

\section{References}

Averill, J. R. (1973). Personal control over aversive stimuli and its relationship to stress. Psychological Bulletin, 80, 286-303. https://doi.org/10.1037/h0034845

Bandura, A. (1977). Self-efficacy: Toward a unifying theory of behavioural change. Psychological Review, 84, 191-215. https://doi.org/10.1016/0146-6402(78)90002-4

DeCharms, R. (1979). Personal causation and perceived control. In L. C. Perlmuter \& R. A. Monty (Eds.), Choice and perceived control (pp. 29-40). Hillsdale, NJ: Erlbaum.

Fock, H., Hui, M. K., Au, K., \& Bond, M. H. (2013). Moderation effects of power distance on the relationship between types of empowerment and employee satisfaction. Journal of Cross-Cultural Psychology, 44(2), 281-298. https://doi.org/10.1177/0022022112443415

Heckhausen, J., \& Schulz, R. (1995). A life-span theory of control. Psychological Reviews, 102, 284-384. https://doi.org/10.1037/0033-295X.102.2.284

Huang, Y., Robertson, M. M., \& Chang, K. (2004). The role of environmental control on environmental satisfaction, communication, and psychological stress: Effects of office ergonomics training. Environment and Behaviour, 36(5), 617-637. https://doi.org/10.1177/0013916503262543

Karasek, R. A. (1979). Job demands, job decision latitude, and mental strain: Implications for job redesign. Administrative Science Quarterly, 24, 285-308. https://doi.org/10.2307/2392498

Kim, H., \& Markus, H. (1999). Deviance or uniqueness, harmony or conformity? A cultural analysis. Journal of Personality and Social Psychology, 77, 785-800. https://doi.org/10.1037/0022-3514.77.4.785

Langston, C., Song, Y., \& Purdey, B. (2008). Perceived conditions of workers in different organisational settings. Facilities, 26(1/2), 54-67. https://doi.org/10.1108/02632770810840309 
Leaman, A. (1995). Dissatisfaction and office productivity. Facilities, 13(2), 13-19. https://doi.org/10.1108/ 02632779510078120

Leaman, A. (2003). User needs and expectations. In R. J. Cole \& R. Lorch (Eds.), Buildings, culture \& environment: Informing local \& global practices (pp. 154-176). Oxford: Blackwell.

Leung, K. (2001). Different carrots for different rabbits: Effects of individualism - Collectivism and power distance on work motivation. In M. Erez, U. Kleinbeck, \& H. Thierry (Eds.), Work motivation in the context of a globalising economy (pp. 329-339). New York: Lawrence Erlbaum Associates Publishers.

McConatha, J. T., \& Huba, H. M. (1999). Primary, secondary, and emotional control across adulthood. Current Psychology, 18(2), 164-170. https://doi.org/10.1007/s12144-999-1025-z

McCoy, J. M., \& Evans, G. W. (2005). Physical work environment. Thousand Oaks, CA: Sage.

Mirowsky, J., \& Ross, C. E. (1990). Control or defense? Depression and the sense of control over good and bad outcomes. Journal of Health and Social Behaviour, 31, 71-86. https://doi.org/10.2307/2137046

Morling, B., \& Evered, S. (2006). Secondary control reviewed and defined. Psychological Bulletin, 132(2), 269-296. https://doi.org/10.1037/0033-2909.132.2.269

O’Neill, M. J. (2010). A model of environmental control and effective work. Facilities, 28(3/4), 118-136. https://doi.org/10.1108/02632771011023104

O'Neill, M. J., \& Carayon, P. (1993). The relationship between privacy, control, and stress responses in office workers. Proceedings of the Human Factors and Ergonomics Society Annual Meeting, 3(3), 479-483. https://doi.org/10.1177/154193129303700702

Peng, K., \& Nisbett, R. E. (1999). Culture, dialectics, and reasoning about contradiction. American Psychologist, 54, 741-754. https://doi.org/10.1037/0003-066X.54.9.741

Piaget, J. (1970). Piaget's theory. In P. H. Mussen (Ed.), Carmicheal's manual of child psychology (3rd ed.). New York: Wiley.

Robertson, M. M., \& O’Neill, M. J. (1999). Effects of environmental control on stress, performance and group effectiveness. Proceedings of the Human Factors and Ergonomics Society Annual Meeting, 43(8), 552-556. https://doi.org/10.1177/154193129904300801

Rothbaum, F., Weisz, J. R., \& Snyder, S. S. (1982). Changing the world and changing the self: A twoprocess model of perception control. Journal of Personality and Social Psychology, 42, 5-37. https://doi. org/10.1037/0022-3514.42.1.5

Rotter, J. B. (1966). Generalised expectancies of internal versus external control of reinforcements. Psychological Monographs, 80(1), 1-28. https://doi.org/10.1037/h0092976

Seligman, M. E. P. (1975). Helplessness: On depression, development and death. San Francisco, CA: W. H. Freeman.

Skinner, E. A. (1996). A guide to constructs of control. Journal of Personality and Social Psychology, 71(3), 549-570. https://doi.org/10.1037/0022-3514.71.3.549

Spector, P. E. (1988). Development of the work locus of control scale. Journal of Occupational Psychology, 61, 335-340. https://doi.org/10.1111/j.2044-8325.1988.tb00470.x

Spector, P. E., Sanchez, J. I., Siu, O. L., Salgado, J., \& Ma, J. (2004). Eastern versus Western control beliefs at work: An investigation of secondary control, socioinstrumental control, and work locus of control in China and the US. Applied Psychology: An International Review, 53, 38-60. https://doi. org/10.1111/j.1464-0597.2004.00160.x

Veitch, J. A., \& Newsham, G. R. (2000). Exercised control, lighting choices, and energy use: An office simulation experiment. Journal of Environmental Psychology, 20(3), 219-237. https://doi.org/10.1006/ jevp.1999.0169

Vischer, J. C. (2008). Towards an environmental psychology of workspace: How people are affected by environments for work. Architectural Science Review, 51(2), 97-108. https://doi.org/10.3763/asre.2008.5114

Weisz, J. R., Rothbaum, F. M., \& Blackburn, T. C. (1984). Standing out and standing in: The psychology of control in America and Japan. American Psychologist, 39, 955-969. https://doi.org/10.1037/0003-06 6X.39.9.955

Xie, D. (2019). Cultural influence in Chinese workspaces: National, regional, industrial and organisational effects [Doctoral thesis]. London: University College London.

Zhang, Y., \& Begley, T. M. (2011). Power distance and its moderating impact on empowerment and team participation. The International Journal of Human Resource Management, 22(17), 3601-3617. https://doi.org/10.1080/09585192.2011.560877 